\title{
Imipramine decreases oesophageal pain perception in human male volunteers
}

\author{
P L Peghini, P O Katz, D O Castell
}

\begin{abstract}
Background-Visceral hyperalgesia is a hallmark of functional gastrointestinal disorders. Antidepressants improve symptoms in these patients, although their mode of action is unclear. Antidepressant, anticholinergic, and analgesic mechanisms have been proposed.
\end{abstract}

Aims-To investigate whether imipramine, which has a visceral analgesic effect, increases pain thresholds to experimental visceral pain.

Methods-Visceral perception for first sensation and pain was measured with intraoesophageal balloon distension in $\mathbf{1 5}$ male volunteers. The effect of imipramine was studied in a double blind, placebo controlled, crossover study. Imipramine was given in ascending doses for 12 days (25 $\mathrm{mg}$ days $1-3,50 \mathrm{mg}$ days $4-6,75 \mathrm{mg}$ days 7-12), with oesophageal perception studied on day 13.

Results-Inflation volumes and intraballoon pressures at first sensation were not different between placebo and imipramine. Balloon inflation volume at pain threshold was higher on imipramine $(p=0.015)$. Median intraballoon pressures were not different at pain threshold for placebo and imipramine. Oesophageal wall compliance was not affected by imipramine.

Conclusion-Increased pain thresholds on imipramine in this group of normal male volunteers in the absence of changes in oesophageal tone imply the presence of a visceral analgesic effect.

(Gut 1998;42:807-813)

Keywords: antidepressants; imipramine; visceral hyperalgesia; oesophageal balloon; distension; functional bowel syndromes

Department of Medicine, Suite 501, Pepper Pavilion, Allegheny University Hospitals, Graduate, One Graduate Plaza, 1800 Lombard Street, Philadelphia,

Pennsylvania 19146, USA

P L Peghini

P O Katz

D O Castell

Correspondence to: Dr D O Castell.

Accepted for publication 19 January 1998
Visceral hyperalgesia is currently believed to play a crucial role in the pathogenesis of functional bowel syndromes. ${ }^{1}$ This concept stems from the observation that patients with unexplained chest pain (UCP), functional dyspepsia, or irritable bowel syndrome (IBS) have reduced tolerance to balloon distension of the respective parts of the gut. ${ }^{2-5}$ The possible presence of generalised visceral hyperalgesia is suggested by several observations: the lower threshold to balloon perception is not restricted to the index organ, but occurs throughout the whole gastrointestinal tract; it is not accompanied by alterations in motility; and it is not associated with increased sensitivity to the perception of somatic stimuli. ${ }^{36-11}$
Antidepressants such as the tricyclic compounds imipramine, desipramine, clomipramine, and amitriptyline and the atypical antidepressant trazodone alleviate chronic pain of somatic and visceral origin. The mechanism of this effect is unknown. Proposed possibilities include a function relating to their antidepressive or anticholinergic properties and a direct analgesic effect. ${ }^{12-24}$

Imipramine decreases pain thresholds to experimental somatic pain in healthy volunteers, suggesting the presence of an analgesic effect. ${ }^{25}$ It also improves symptoms in patients with UCP. ${ }^{19}$ This study was designed to determine whether the improvement of visceral symptoms is mediated through a visceral analgesic effect. We hypothesised that imipramine would increase pain thresholds to intraoesophageal balloon distension (IOBD) in normal volunteers.

\section{Methods}

SUBJECTS

Fifteen healthy male volunteers, median age 36 years (range 21-45), without history of dysphagia or heartburn, were studied.

\section{EXPERIMENTAL PROTOCOL}

The effect of imipramine on oesophageal sensory perception was assessed in a randomised, double blind, placebo controlled, crossover trial. Subjects were randomised by blindly taking a number from a closed container and given identical capsules in a pill box with written instructions to take them for 12 days at bedtime. Eight subjects received imipramine first and seven received placebo first. On the morning of day 13, while fasting, sensation and pain thresholds were assessed with IOBD. The capsules contained either placebo or $25 \mathrm{mg}$ imipramine (Biocraft Laboratories, Elmwood Park, New Jersey). According to the instructions the dosing was one capsule at bedtime for three days, followed by two capsules for three days, followed by three capsules for six days. After testing on day 13, subjects were crossed over to the other treatment and the same regimen was repeated, terminating with the second IOBD 13 days later. One subject failed to increase to $75 \mathrm{mg}$ of imipramine due to urinary hesitancy and remained on the $50 \mathrm{mg}$ dose for a total of nine days. The investigator performing the balloon testing was not aware of this.

INTRAOESOPHAGEAL BALLOON DISTENSION The technique used has been described previously. ${ }^{26}$ The balloon catheter consisted of a $14 \mathrm{Fr}$ plastic catheter with a $45 \mathrm{~mm}$ long latex 


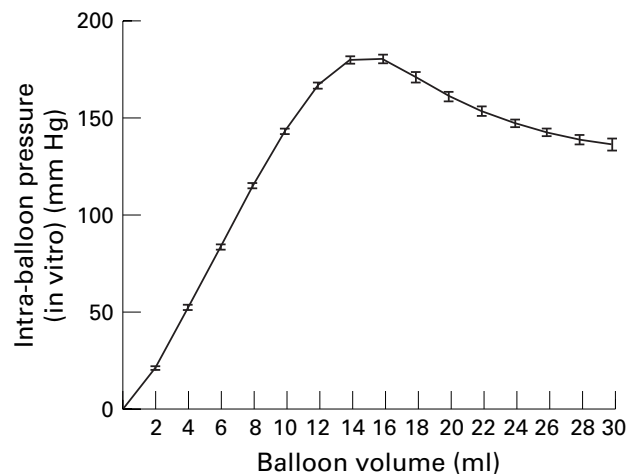

Figure 1 Balloon compliance. Mean pressure volume curve of the 30 pre-IOBD, in vitro assessments of the six balloons used in the study.

balloon located $10 \mathrm{~cm}$ from the distal end (Wilson-Cook Medical Inc., Winston-Salem, North Carolina, USA). It was inserted transnasally without application of local anaesthetic and the balloon was placed with the centre at $10 \mathrm{~cm}$ above the lower oesophageal sphincter (LOS). The position of the LOS had been measured manometrically on a prior screening visit. Using a special air delivery system (Wilson-Cook) the balloon was inflated at a rate of $170 \mathrm{ml}$ per second, remained inflated for two seconds (dwell time), and was then deflated quickly. As in our previous studies, inflations were started at a volume of $2 \mathrm{ml}$ and increased in steps of $2 \mathrm{ml}$ up to one step above the occurrence of chest pain or until the maximum inflation of $30 \mathrm{ml}$. We believe that continuing to one step beyond occurrence of chest pain enhances the quality of our assessment - that is, it provides proof that the pain threshold reached was really the pain threshold. This proved to be the case since all subjects had pain at the inflation one step above pain threshold. Balloon distensions were done in end inspiration. Subjects were instructed to respond to each inflation by indicating whether they felt "no sensation", "sensation in the chest", or "chest pain". Sham inflations were performed with the pump running and the balloon disconnected from the pump. One was done two to four inflations after reaching the threshold for sensation, and a second was done after reaching the pain threshold. After a rest of 10 minutes this series



Figure 2 Relation between balloon volume, pressure, and diameter in vitro.
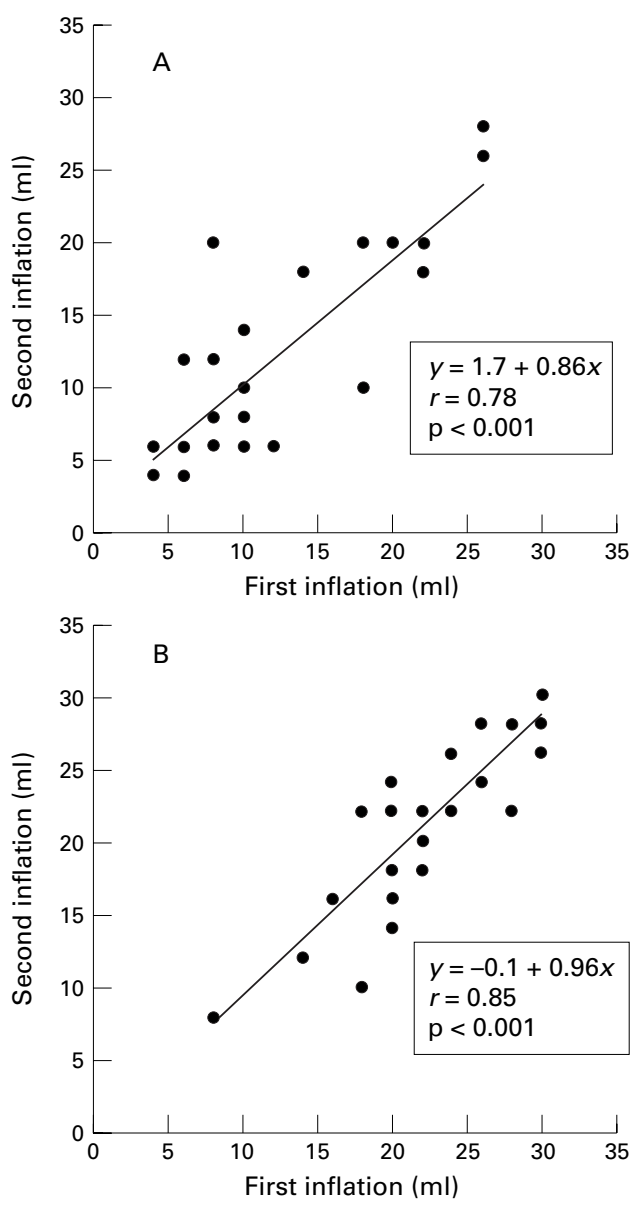

Figure 3 Reproducibility of perception thresholds of the two testing days. Volumes at perception threshold for first inflation series ( $x$ axis) plotted against volumes at perception threshold for second inflation series 10 minutes later (y axis). (A) Sensation thresholds; (B) pain thresholds.

of inflations was repeated. The pressure in the tubing system was constantly displayed to exclude leakage (Universal Pressure Meter, Biotech Instruments Inc., Vermont, USA) and plateau pressures were recorded for each inflation. Pressure volume characteristics of the balloon were assessed before each study on the bench (in vitro) and balloons with increased compliance were discarded. Six balloons were used for the study which took eight months to complete. A total of 30 IOBDs (each of the 15 subjects was tested twice), were performed during this time. Figure 1 shows the mean pressure volume curve of the 30 in vitro assessments of the six balloons used for IOBD. The small standard errors indicate that the balloons used in this study had comparable and stable compliance. Figure 2 shows the behaviour of balloon pressure and diameter in relation to inflation volume in vitro.

Only after completion of the entire study, at the end of the second IOBD, were the subjects asked whether they noticed any symptoms on either of the two treatments. The study was intentionally not designed to assess side effects on imipramine because a high incidence was expected and every attempt was made to avoid bias of the subjects and investigators. Possible side effects were mentioned only during the 

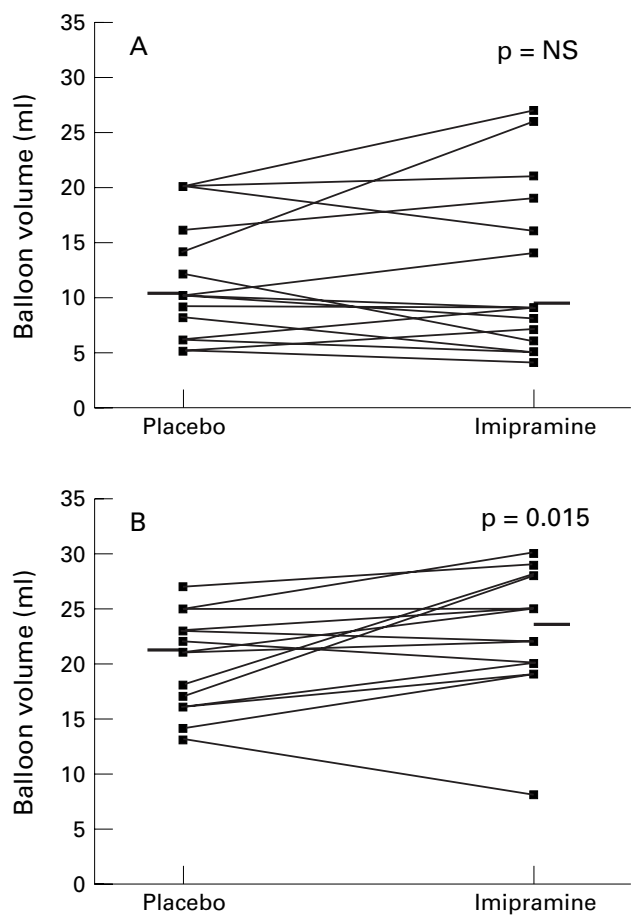

Figure 4 Perception thresholds on placebo and imipramine. (A) Volumes at thresholds for first sensation $(n=15) ;(B)$ volumes at pain thresholds $(n=14)$. Median represented by horizontal bar.
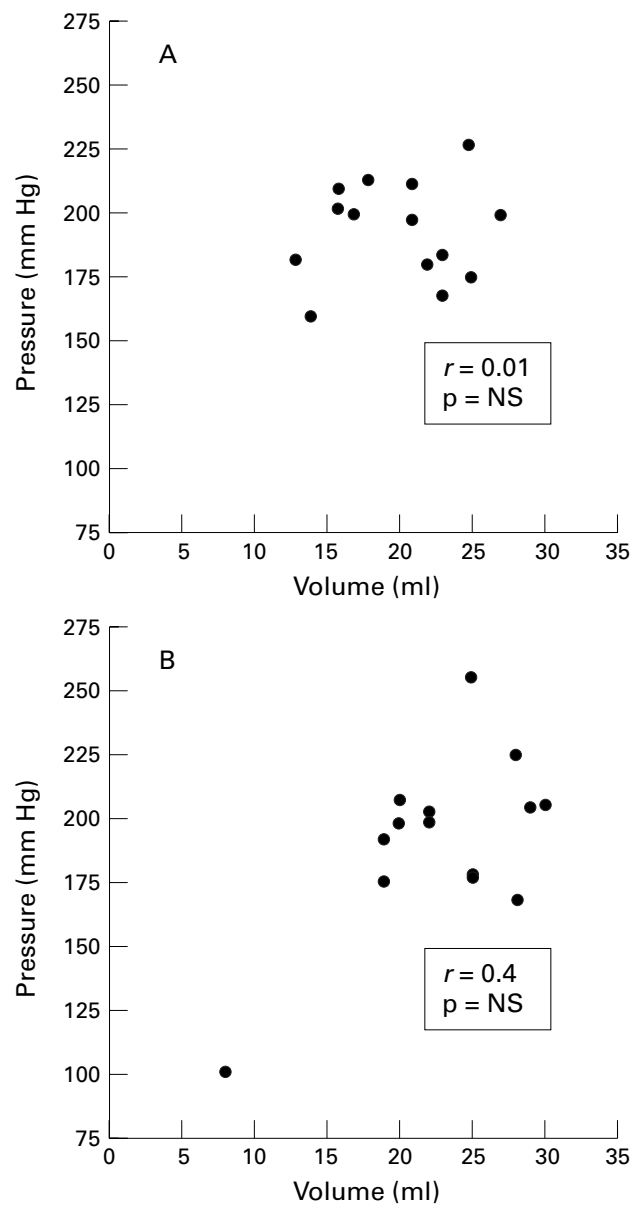

Figure 5 Balloon volume and pressure at pain threshold for placebo $(A)$ and imipramine $(B)$. consent procedure. Active and passive recollection of symptoms was then recorded after completion of the study only, recognising the potential that symptoms occurring on the first treatment might be under-reported.

REPRODUCIBILITY OF PERCEPTION THRESHOLDS The reproducibility of the measurements of perception thresholds with IOBD was assessed by comparing the volumes at perception threshold of the first with the second inflation series (10 minutes later). Comparison was done by calculating the correlation and regression characteristics between the first and the second assessment of both test days of all subjects. The total number of observations was 28 for pain thresholds because one subject experienced no pain at maximum balloon inflation of $30 \mathrm{ml}$. The total number was 29 for sensation thresholds because on one occasion one subject went directly from "no sensation in the chest" to "chest pain". Absolute reproducibility would yield a regression coefficient and slope of the regression line of 1.0, and the y intercept would be zero. Actual values for sensation thresholds were: correlation coefficient $0.78(p<0.001)$, slope 0.86 , and y intercept 1.7, indicating good reproducibility (fig 3A). Excellent reproducibility was found for pain thresholds: correlation coefficient 0.85 $(\mathrm{p}<0.001)$, slope 0.96 , and $y$ intercept -0.1 (fig 3B). This effect has previously been identified, both in our laboratory ${ }^{26} 27$ and elsewhere. ${ }^{28} 29$

DATA ANALYSIS AND STATISTICS

Individual averages of volumes and intraballoon pressures at sensation and pain threshold for the two inflation series were used for analysis. Group medians for perception thresholds on placebo and imipramine were compared with Wilcoxon's signed ranks test. Correlations between balloon volumes and balloon pressures were calculated with Spearman's rank correlation. Categorical data were compared with Fisher's exact test. Probability values for comparison of perception thresholds are one tailed, all others are two tailed. Statistical software used was True Epista, fourth edition (Epistat Services, Richardson, Texas, USA). Oesophageal wall compliance was assessed by plotting the differences of the in vivo minus the in vitro intraballoon pressures (group means $(\mathrm{SEM})$ ) against the corresponding inflation volumes.

The protocol was approved by the Institutional Review Board of the Graduate Hospital.

\section{Results}

EFFECT OF IMIPRAMINE ON PERCEPTION THRESHOLDS

Sensation

The median inflation volume at threshold for sensation did not differ between the two treatments: it was $10 \mathrm{ml}$ (interquartile range (IQR) 7-15 ml) for placebo and $9 \mathrm{ml}$ (IQR 6.5-17.5 $\mathrm{ml}$ ) for imipramine (NS). Seven subjects had a higher threshold with imipramine, seven with placebo, and one had the same threshold on both treatments (fig $4 \mathrm{~A}$ ). There was also no change in median intraballoon pressure at 


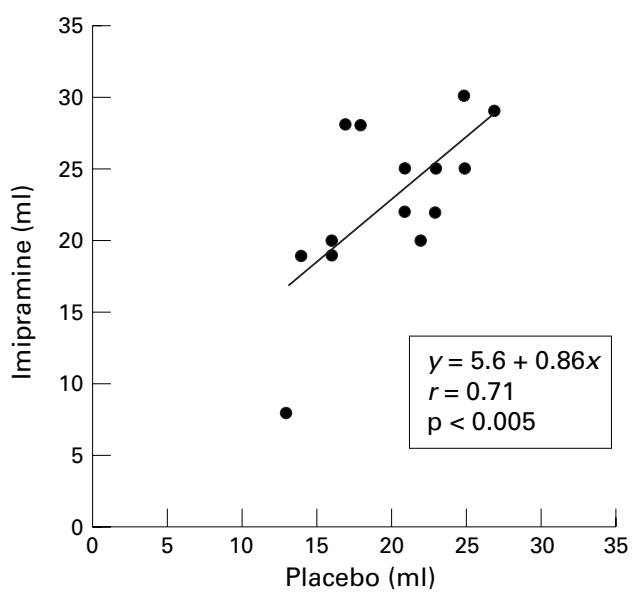

Figure 6 Volumes at pain threshold on placebo and imipramine.

sensation thresholds, $138 \mathrm{~mm} \mathrm{Hg}$ (IQR 82.25$173.25 \mathrm{~mm} \mathrm{Hg}$ ) for placebo versus $133 \mathrm{~mm} \mathrm{Hg}$ (IQR 78-202 $\mathrm{mm} \mathrm{Hg}$ ) for imipramine (NS).

Pain

The balloon inflation volume at pain threshold was significantly higher on imipramine than on placebo. Median values were $23.5 \mathrm{ml}$ (IQR 20-27.3 ml) for imipramine and $21 \mathrm{ml}$ (IQR 16.3-23 ml) for placebo $(\mathrm{p}=0.015)$. One subject experienced no pain on either placebo or imipramine at maximum inflation of $30 \mathrm{ml}$. The calculation of pain thresholds is therefore based on only 14 observations. One subject had no pain at maximum inflation while on imipramine. A pain threshold of $30 \mathrm{ml}$ was assumed for statistical purposes. Ten volunteers had an increase, three a decrease, and one no change in pain threshold (fig 4B). Median intraballoon pressures at pain threshold were not different between the two treatments, $198.25 \mathrm{~mm} \mathrm{Hg}$ (IQR $180.5-207.5 \mathrm{~mm} \mathrm{Hg}$ ) for placebo versus $199.25 \mathrm{~mm} \mathrm{Hg}$ (IQR 178.25$205.75 \mathrm{~mm} \mathrm{Hg}$ ) for imipramine (NS). Balloon volume and balloon pressure at pain threshold

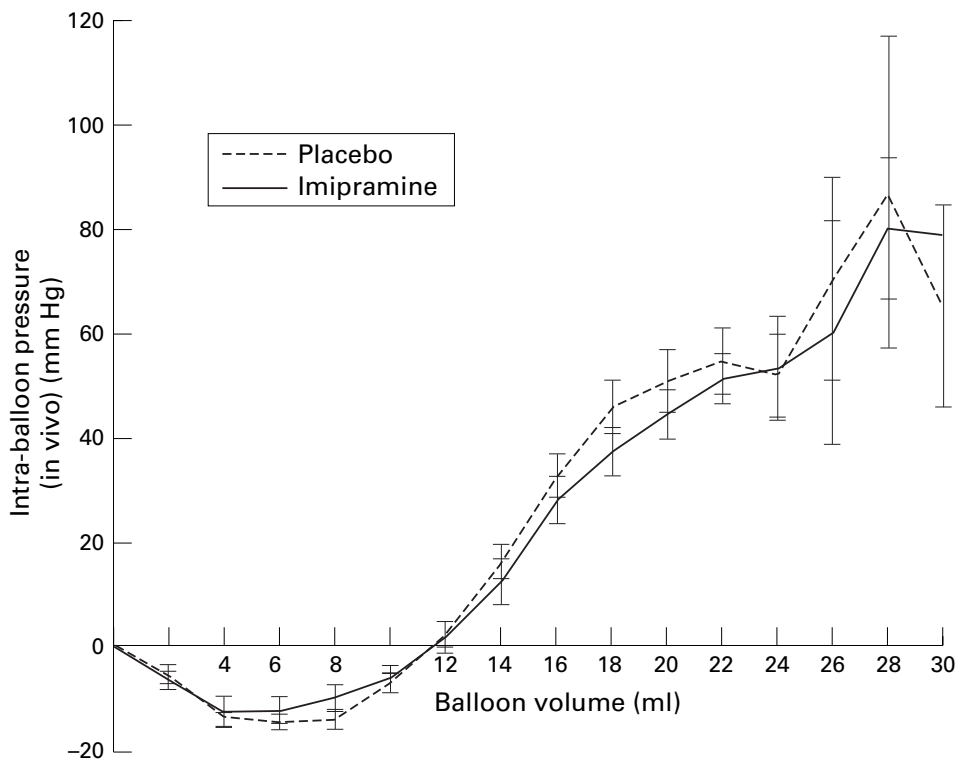

Figure 7 Oesophageal wall compliance. Difference between in vivo minus in vitro balloon pressure (mean (SEM)) at corresponding inflation volumes. were poorly correlated for both treatments $(r<0.5$, NS for both) (fig 5).

\section{SHAM INFLATIONS}

A total of 57 sham inflations were done. Thirty were applied after reaching the threshold for sensation and 27 after reaching the pain threshold. Five sham inflations (9\%) were associated with a perception in the chest. Three of these were performed after reaching the threshold for sensation and two after reaching the pain threshold. All five were rated as "sensation"; none was rated as "pain".

All real inflations above the pain threshold were rated as painful.

CORRELATION OF VOLUMES AT PAIN THRESHOLD ON IMIPRAMINE VERSUS PLACEBO

Pain thresholds on imipramine for the individual subjects showed a good correlation with those on placebo $(r=0.71, \mathrm{p}<0.005)$. The regression line follows the equation $\mathrm{y}=5.6+$ $0.86 \mathrm{x}$ (fig 6 ). The highly significant correlation and the slope close to $1(0.86)$ would seem to indicate that the magnitude of change in the pain threshold of each individual is a reflection of their baseline - that is, the placebo threshold. The y intercept of 5.6 roughly defines the expected median increase in pain threshold.

OESOPHAGEAL WALL COMPLIANCE

The compliance curves for placebo and imipramine were not different, suggesting no effect of imipramine on oesophageal tone (fig 7 ). The negative values at low inflation volumes are due to the fact that inflations were performed at end inspiration, and reflect the negative intrathoracic pressure. Because inflations were stopped one step after the occurrence of pain, the number of measurements decreased at higher inflation volumes. This explains the increasing standard errors at inflation volumes above $22 \mathrm{ml}$.

\section{SIDE EFFECTS}

All volunteers completed the study. Eleven subjects noticed symptoms on imipramine only and one had symptoms on placebo only (happiness). One subject had symptoms on both treatments (constipation on placebo) and two had no symptoms on either treatment. The relative risk of developing a symptom on imipramine was 4.9 (95\% confidence interval 1.7 to 13.8$)(p<0.001)$. Of the 12 subjects with symptoms on imipramine, eight $(67 \%)$ had at least one anticholinergic symptom: xerostomia and constipation each in five subjects, erectile dysfunction and urinary hesitancy each in one subject. Seven of these 12 subjects had one or more central nervous symptoms such as insomnia in two subjects, tiredness in three subjects, irritability in one subject, and happiness in three subjects.

\section{Discussion}

This study shows that imipramine increases the pain threshold to IOBD in normal volunteers. It is to our knowledge the first report 
documenting an analgesic effect of a tricyclic antidepressant on experimental visceral pain in normal volunteers.

Placebo controlled studies of antidepressants have shown that they provide symptomatic relief in chronic pain conditions of somatic and visceral origin, including diabetic neuropathy, postherpetic neuralgia, UCP, and IBS. The mechanism of this effect is not known. Possibilities that have been proposed include an effect based on their antidepressant or anticholinergic properties or a direct analgesic action. ${ }^{12-24}$ Symptoms of depression are highly prevalent among patients with chronic pain, making this cohort susceptible to the effects of an antidepressant. ${ }^{17}$ The favourable response to antidepressants in chronic pain, however, is not believed to be mediated through improvement of depression for a number of reasons. Firstly, serum concentrations required to reduce pain are lower than those needed to treat mood disorders: $100-120$ $\mu \mathrm{g} / 1$ compared with $150-300 \mu \mathrm{g} / 1 \mathrm{respectively}$ for imipramine (including its first metabolite desipramine). Secondly, pain relief occurs earlier (after four days to two weeks) than improvement of symptoms of depression, which occurs after two to four weeks. ${ }^{14} 1720$ 30-32 Thirdly, antidepressants have analgesic properties in patients without signs of depression, and analgesia occurs without concurrent improvement of mood status in patients with pain. ${ }^{121618} 28$ The ability of antidepressants to reduce the response to painful stimuli in animals furthermore supports an analgesic mechanism..$^{34}$

The most compelling evidence for the presence of an analgesic mechanism comes from a report by Bromm et $a l^{25}$ who studied the effect of imipramine on experimental somatic pain induced by intracutaneous electrical shocks in normal volunteers. A dose of $100 \mathrm{mg}$ given orally decreased pain thresholds, pain ratings, amplitudes of pain related somatosensory evoked cerebral potentials, and alpha activity of the electroencephalogram to a degree comparable with that of one dose of oral meperidine $(150 \mathrm{mg})$, although with a delayed onset. $^{25}$

In the present study we performed an analogous experiment in a model of visceral pain. The increment in pain threshold was confirmed in the oesophagus. Although the median increase was only $12 \%$, a relatively small increase in pain threshold may provide substantial clinical improvement. Our volunteers were studied eight to 12 hours after intake of the last dose of imipramine. In Bromm et al's report the maximum analgesic effect of imipramine appeared about two hours after intake of the drug, coincident with expected peak plasma concentrations. ${ }^{25}$ One can therefore speculate that a greater effect might have been shown if we had given the dose closer to the testing. Likewise, would patients being treated for pain be more likely to benefit from dosing in the morning or twice a day? Our decision to give the drug at bedtime was based on the prior observation that this regimen caused symptomatic relief in patients with UCP, and on standard recommendations. ${ }^{19} 32$ The latter, however, apply to the treatment of depression which necessitates higher doses than those needed to treat pain and reflect an attempt to minimise side effects. Nevertheless, side effects were present in the majority of the young healthy volunteers studied in this report even with relatively low doses given at bedtime. Although the symptoms were generally rated mild, they necessitated dose reduction in one case and they might be a more limiting factor for dose administration during the day.

The high incidence of side effects consistent with the anticholinergic properties of imipramine raises the question of whether the observed increase in pain thresholds is due to the latter. This question has been addressed in two studies, in which the effect of atropine on oesophageal sensory thresholds was assessed with IOBD. In the first, atropine was associated with a decrease in mean pain score in volunteers. The interpretation of these data, however, is complicated by the fact that this decrease resulted from a high baseline score prior to atropine. The post atropine score and the pre and post placebo scores were almost equal. ${ }^{35}$ In the second study atropine had no effect on pain thresholds in volunteers and patients with UCP. This study was not primarily designed to assess the effect of atropine on oesophageal pain thresholds, since the drug was administered to block the effect of edrophonium given 30 minutes previously. Nevertheless, it argues against an alteration of oesophageal perception by atropine, because the short half life of edrophonium of about five minutes makes it unlikely that it affected the testing done with atropine 30 minutes later. ${ }^{86}$

The mechanism by which an anticholinergic drug could affect sensation would most likely be through alteration of the motor limb of the nervous system, as the cholinergic system has no role in the sensory limb. ${ }^{37} 38$ Because the response of hollow viscera to distension depends on the compliance of the organ, which in turn depends on smooth muscle tone, sensory perception could be affected by anticholinergic mechanisms through changes in compliance. ${ }^{9}$ The oesophageal compliance, however, was not affected by imipramine, indicating that the dose given in this study was not high enough to affect oesophageal tone (fig 7). In a recent report in patients with UCP, atropine given in doses high enough to decrease oesophageal tone caused a decrease rather than the expected increase in oesophageal pain threshold. ${ }^{39}$ Taken together, it seems unlikely that the observed increase in pain threshold caused by imipramine is due to the anticholinergic properties of the drug.

Thresholds for sensation were not affected by imipramine. The good reproducibility of sensation thresholds suggests that this is a real finding (fig 3A). This result is in keeping with that of Bromm et al who also found no alteration of sensation threshold with imipramine in somatic pain. ${ }^{25}$ In addition, there is convincing evidence from animal studies that the pain modulating effect of antidepressants relies on potentiation of central, opioid mediated 
descending, analgesic pathways. ${ }^{33} 340$ An alteration of sensation would therefore be unexpected.

The high frequency of side effects on imipramine carries the risk of unblinding of the volunteers, with consecutive bias during IOBD. This is a flaw inherent in all studies using antidepressants with anticholinergic type side effects, unless they are compared with atropine. However, this problem is often not acknowledged in such studies. ${ }^{1925}$ Our volunteers knew only that visceral perception was being tested, without having knowledge about the hypothesised direction of the change. Whether it can be assumed that these expectations evened out statistically is difficult to assess.

The increment in pain threshold with imipramine was only seen with volume at pain threshold but not with pressure at pain threshold, and there was no correlation between the two (fig 5). Although volume is used most often in studies of this type, opinions as to which parameter is more appropriate differ. ${ }^{41}{ }^{42}$ The answer to this question, as to many others pertaining to IOBD, may depend on the technique used, as inflation protocol, location, and composition of the balloon affect perception thresholds to IOBD and their response to interventions such as acid perfusion of the oesophagus. ${ }^{43}$ The oesophagus, unlike the stomach or colon, is not designed to function as a reservoir. This has some bearing on the inflation protocol. Luminal distension triggers secondary peristalsis, aimed at propelling the bolus into the stomach. ${ }^{44}$ According to our experience, and that of others, these contractions can cause strong aborad forces producing discomfort at the nose, where the balloon catheter is fixed. ${ }^{8}$ This not only distracts the subject, but could theoretically also generate sensations in the chest. With a dwell time of only two seconds and complete deflations between inflations this problem was essentially avoided in the present study. Balloon distension causes a phasic stimulus during inflation and a tonic stimulus while the balloon is inflated. It is important to define which stimulus is being tested, because the two can be rated differently and their response to pharmacological interventions can differ. ${ }^{45}$ Pressures reported in this study were read during the plateau phase of the inflation cycle, and therefore reflect a parameter of the tonic phase of the stimulus. As the inflation velocity of the pump was stable, changes in inflation volume resulted in changes in the duration of the inflation-that is, the duration of the phasic stimulus. Inflation volume can therefore be considered primarily a parameter affecting the phasic stimulus. The absence of a difference of pressures at pain threshold could be explained by two scenarios. Firstly, imipramine could affect only phasic but not tonic pain. This would be in accordance with the fact that the analgesic effect of imipramine in somatic pain was shown using a phasic pain stimulus. ${ }^{25}$ The observation that desipramine, given in a dose able to cause symptomatic improvement in patients with functional dyspepsia, did not increase gastric sensory thresholds assessed using the barostat technique could also be explained by this argument. ${ }^{24} \mathrm{~A}$ second explanation of why pressures were not different would be that the combination of rapid inflation with short dwell time resulted mainly in an assessment of phasic pain, lacking the sensitivity needed to detect a change in tonic pain thresholds.

In our studies, distension stimuli were applied in an ascending series. This protocol has been criticised because subjects can recognise the inflation pattern and the anticipation of stimuli of greater intensity can not only bias the response according to factors other than pain perception, such as level of anxiety or vigilance, but can also cause sham inflations to be perceived as painful. ${ }^{45}$ Although it is important to eliminate cues with the potential to affect the perception and reporting of pain differently in the groups being compared, for example patients and volunteers, this should be less problematic in a crossover study. In fact, we have shown in the past that sequential and random IOBD yield the same results if assessed in such a manner. ${ }^{26}$ The low rate of sham inflations accompanied by perceptions in the chest in our study and the fact that none of them was rated as painful suggest that anticipation did not play a major role in the assessment of pain thresholds in our study.

Our results are not consistent with the prior report by Cannon et al. ${ }^{19}$ In that study patients with UCP treated with imipramine had no change in pain thresholds to IOBD despite clinical improvement. Three reasons could account for this discrepancy. Firstly, patients were given $50 \mathrm{mg}$ of imipramine compared with the $75 \mathrm{mg}$ used in our study. Secondly, topical lidocaine was applied for the insertion of the balloon. This could have impaired the ability to perceive the balloon distensions. The application of local anaesthetic to the oesophageal mucosa has been shown to decrease oesophageal perception. ${ }^{47}$ Thirdly, a beta error is possible because the study had no crossover design and included only seven observations, as reproduction of the typical chest pain was sought. It seems therefore justified to question the negative result of the IOBD in that study, particularly since the other assessment of visceral pain, cardiac pacing, did show an increase in pain tolerance with imipramine. ${ }^{19}$

In summary, we have shown that imipramine increases pain thresholds to IOBD in normal male volunteers. The absence of psychiatric disease and the absence of a change in oesophageal compliance with imipramine argue against our results being based on the antidepressant or anticholinergic properties of the drug and suggest a visceral analgesic effect.

This work was supported by a grant from the American College of Gastroenterology, Institute for Research, and from the TheoZürich, Switzerland. It was presented at the annual meeting of the American Gastroenterological Association, Washington DC, May 1997.

\footnotetext{
1 Mayer EA, Gebhart GF. Basic and clinical aspects of visceral hyperalgesia. Gastroenterology 1994;107:271-93.

2 Barish CF, Castell DO, Richter JE. Graded oesophageal Barish CF, Castell DO, Richter JE. Graded oesophageal
balloon distention: a new provocative test for noncardiac chest pain. Dig Dis Sci 1986;31:1292-8.
} 
3 Richter JE, Barish CF, Castell DO. Abnormal sensory perception in patients with oesophageal chest pain. Gastropertory 1986;91:845-52.

4 Lémann M, Dederding JP, Flourié B, et al. Abnormal perception of visceral pain in response to gastric distension in chronic idiopathic dyspepsia - the irritable stomach syndrome. Dig Dis Sci 1991;36:1249-54

5 Ritchie J. Pain from distension of the pelvic colon by inflating a balloon in the irritable colon syndrome. Gut 1973;14 125-32.

6 Costantini M, Sturniolo GC, Zaninotto G, et al. Altered oesophageal pain threshold in irritable bowel syndrome. Dig Dis Sci 1993;38:206-12.

7 Trimble KC, Farouk R, Pryde A, et al. Heightened visceral sensation in functional gastrointestinal disease is not site specific: evidence for a generalized disorder of gut sensitivity. Dig Dis Sci 1995;40:1607-13.

8 De Caestecker JS, Pryde A, Heading RC. Site and mechanism of pain perception with oesophageal balloon distention and intravenous edrophonium in patients with distention and intravenous edrophon: chest pain. Gut 1992;33:580-6.

9 Mayer EA, Raybould HE. Role of visceral afferent mechanisms in functional bowel disorders. Gastroenterology

10 Cook IJ, van Eeden A, Collins SM. Patients with irritable bowel syndrome have a greater pain tolerance than normal subjects. Gastroenterology 1987;93:727-33.

11 Whitehead WE, Holtkotter B, Enck P, et al. Tolerance for rectosigmoid distention in irritable bowel syndrome. Gastroenterology 1990;98:1187-92.

12 Egbunike IG, Chaffee BJ. Antidepressants in the management of chronic pain syndromes. Pharmacotherapy 1990 10:262-70.

13 Max MB, Lynch SA, Muir J, et al. Effects of desipramine, amitriptyline and fluoxetine on pain in diabetic neuropathy. N Engl f Med 1992;326:1250-6.

14 Kvinesdal B, Molin J, Froland A, et al. Imipramine treatment of painful diabetic neuropathy. FAMA 1984;251: treatment

15 Sindrup SH, Brosen K, Gram LF. Antidepressants in pain treatment: antidepressant or analgesic effect? Clin Neurotreatment: antidepressant or

16 Feinman C. Pain relief by antidepressants: possible modes of action. Pain 1985;23:1-8.

17 France RD, Houpt JL, Ellinwood EH. Therapeutic effects of antidepressants in chronic pain. Gen Hosp Psychiatry 1984;6:55-63.

18 Magni G. The use of antidepressants in the treatment of chronic pain. Drugs 1991;42:730-48.

19 Cannon RO, Quyyumi AA, Mincemoyer R, et al. Imipramine in patients with chest pain despite normal coronary angiograms. $\mathrm{N} \mathrm{Engl} \mathrm{f} \mathrm{Med} \mathrm{1994;330:1411-17.}$

20 Clouse RE, Lustman PJ, Geisman RA, et al. Antidepressant therapy in 138 patients with irritable bowel syndrome: a five-year clinical experience. Aliment Pharmacol Ther 1994; 8:409-16.

21 Sindrup SH, Brosen K, Gram LF. The mechanism of action of antidepressants in pain treatment: controlled cross-over studies in diabetic neuropathy. Clin Neuropharmacol studies in diabetic

22 Clouse RE, Lustman PJ, Eckert TC, et al. Low-dose trazodone for symptomatic patients with oesophageal contraction abnormalities. Gastroenterology 1987;92:1027-36.

23 Greenbaum DS, Mayle JE, Vanegeren LE, et al. Effects of desipramine on irritable bowel syndrome compared with atropine and placebo. Dig Dis Sci 1987;32:257-66.

24 Mertz HR, Fass R, Hirsh T, et al. Amitriptyline for functional dyspepsia: effect on symptoms, gastric sensitivity and sleep [abstract]. Gastroenterology 1995;108:A649.
25 Bromm B, Meier W, Scharein E. Imipramine reduces experimental pain. Pain 1986;25:245-57.

26 Lasch H, DeVault KR, Castell DO. Intraesophageal balloon distention in the evaluation of sensory thresholds: studies on reproducibility and comparison of balloon composition. Am f Gastroenterol 1994;89:1185-90.

27 DeVault KR, Castell DO. Esophageal balloon distention and cerebral evoked potential recording in the evaluation of unexplained chest pain. Am f Med 1992;92(suppl 5A): 20S-26S.

28 Barlow JD, Thompson DG. Reproducibility of perception to ooesophageal distension. Gut 1994;35(suppl 2):S8

29 Hazan S, Steinberg A, Morris N, et al. Long-term reproducibility of intraoesophageal balloon distention [abstract]. Gastroenterology 1997;112:A145.

30 Sato TL, Madakasira S. Monitoring tricyclic antidepressant plasma levels. Am Fam Physician 1984;29:199-202.

31 Montastruc JL, Tran MA, Blanc M, et al. Measurement of plasma levels of clomiparmine in the treatment of chronic pain. Clin Neuropharmacol 1985;8:78-82.

32 Baldessarini RJ. Drugs and the treatment of psychiatric disorders. In: Goodman and Gilman, eds. The pharmacological basis of therapeutics. 8th edn. New York: Pergamon Press, 1990:405-23.

33 Botney M, Fields H. Amitriptyline potentiates morphine analgesia by a direct action on the CNS. Ann Neurol 1983; 13:160-4

34 Isenberg KE, Cicero TJ. Possible involvement of opiate receptors in the pharmacological profiles of antidepressant compounds. Eur 7 Pharmacol 1984;103:57-63.

35 Paterson WG, Selucky M, Hynna-Liepert TT. Effect of intraesophageal location and muscarinic blockade on balloon distension-induced chest pain. Dig Dis Sci 1991;36: 282-8.

36 Drachman DB. Myasthenia gravis. In: Isselbacher KJ, et al, eds. Harrison's principles of internal medicine. 13th edn. New York: McGraw-Hill, 1995:2393-6.

37 Lynn RB. Mechanisms of oesophageal pain. Am 7 Med 1992;92 (suppl 5A):11S-19S.

38 Bueno L, Fioramonti J, Delvaux M, et al. Mediators and pharmacology of visceral sensitivity: from basic to clinical investigations. Gastroenterology 1997;112:1714-43.

39 Rao SSC, Hayek B, Summers RW. Unexplained chest pain: oesophageal hypersensitivity or motor dysfunction? [abstract]. Gastroenterology 1997;112:A810.

40 Wang JK. Antinociceptive effect of intrathecally administered serotonin. Anesthesiology 1977;47:269-71.

41 Rao SSC, Gregersen H, Hayek B, et al. Unexplained chest pain: the hypersensitive, hyperreactive, and poorly compliant esophagus. Ann Intern Med 1996:124:950-8.

42 Fass R. Unexplained noncardiac chest pain [letter]. Ann Intern Med 1997;126:662.

43 Peghini PL, Castell DO. Does sensitization of esophageal mucosal receptors occur during provocative testing? $\mathrm{Am} \mathcal{F}$ Gastroenterol 1997;92:919-20.

44 Kahrilas PJ. Functional anatomy and physiology of the esophagus. In: Castell DO, ed. The esophagus 2nd edn. Boston: Little, Brown, 1995:1-28.

45 Naliboff B, Mayer EA. Sensational developments in the irritable bowel. Gut 1996;39:770-1.

46 Silverman DHS, Munakata JA, Ennes H, et al. Regional cerebral activity in normal and pathological perception of visceral pain. Gastroenterology 1997;112:64-72.

47 Becker K, Kuhlbusch R, Enck P, et al. Effect of topical anaesthesia on oesophageal sensory and motor function in healthy subjects. Neurogastroenterol Motil 1994;6:255-61. 